\title{
AN APPROXIMATION PROPERTY CHARACTERIZES ORDERED VECTOR SPACES WITH LATTICE-ORDERED DUALS
}

\author{
BY BERTRAM WALSH ${ }^{1}$ \\ Communicated by François Treves, April 29, 1974
}

This note announces that a simple approximation property, known to hold in large classes of partially ordered locally convex spaces whose duals are lattice ordered (e.g., [Banach or] Fréchet spaces with closed, normal, generating positive cones possessing the Riesz decomposition property), actually characterizes spaces with lattice-ordered duals. Some very mild and natural assumptions about the relation of the topology and the order have to be made, but these are automatically satisfied in Fréchet spaces with closed normal generating cones. This characterization has important consequences in the duality theory of certain classes of linear operators and in questions regarding approximation of harmonic functions; those consequences will be taken up elsewhere.

Let $E[\Sigma]$ be a locally convex space, let $E^{\prime}$ be its dual, and let $K$ be a closed wedge in $E$, i.e., a closed convex set carried into itself under multiplication by nonnegative scalars; let $K^{\prime}=-K^{0}$ denote the wedge dual to $K$. (In the interesting cases $K$ will be a cone.) $K$ determines a wedge $L(E)^{+}$in the algebra $L(E)$ of continuous linear transformations of $E$ into itself, $L(E)^{+}$ consisting of those transformations that carry $K$ into itself. We shall indicate that an element of one of these spaces belongs to the corresponding wedge by saying that the element is "nonnegative" or " $\geqslant 0$ ".

The tensor product space $E^{\prime} \otimes E$ is isomorphic to the ideal in $L(E)$ consisting of linear transformations of finite rank; if $\mathrm{t}=\Sigma x_{i}^{\prime} \otimes x_{i} \in E^{\prime} \otimes E$, the corresponding linear transformation is $T_{t}=\left(x \rightarrow \Sigma\left\langle x, x_{i}^{\prime}\right\rangle x_{i}\right)$ (sums indicated in this way are understood to be finite). Abusing notation slightly, let $K^{\prime} \otimes K=\left\{\Sigma x_{i}^{\prime} \otimes x_{i}: x_{i} \in K, x_{i}^{\prime} \in K^{\prime}\right\} ;$ it is clear that $K^{\prime} \otimes K$ is a wedge. Let $P=\left\{T_{p}: \not \in \in K^{\prime} \otimes K\right\}$.

AMS (MOS) subject classifications (1970). Primary 46A40; Secondary 46M05, 47B55, $3 ! \mathrm{B10}$.

${ }^{1}$ The author was partially supported by NSF-GP-28328.

Copyright $\odot 1974$, American Mathematical Society 
Proposition 1. The following two conditions are equivalent:

If $t \in E^{\prime} \otimes E$ and $T_{. t} \in L(E)^{+}$, then $\operatorname{tr}(t) \geqslant 0$ ("posi-

tive operators have positive traces");

The identity transformation $I \in L(E)$ belongs to the closure of $P$ in the topology of pointwise convergence ("strong operator topology").

THEOREM. Let $E$ satisfy the following hypotheses:

(a) $E^{\prime}=K^{\prime}-K^{\prime}$ ( $K$ is weakly normal [5, p. 220]);

(b) For each $₹$-zero-neighborhood $V \subseteq E$, the set $(V \cap K)-(V \cap K)$

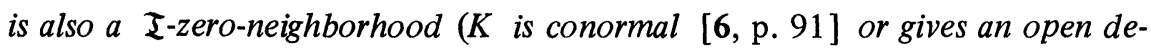
composition [4, p. 94 ff.]).

Then the equivalent conditions (1) and (2) of Proposition 1 above imply that

$$
E^{\prime} \text { is lattice ordered by } K^{\prime} \text {. }
$$

REMARK. Evidently conditions (1) and (2) force the linear hulls $K-K$ and $K^{\prime}-K^{\prime}$ of the respective wedges to be (weakly) dense in $E$ and $E^{\prime}$ respectively. Moreover, $K$ and $K^{\prime}$ must be (proper) cones if these conditions hold, since then for each $x \in K$ there must exist $x^{\prime} \in K^{\prime}$ with $\left.\left\langle x, x^{\prime}\right\rangle\right\rangle$ 0 (and symmetrically). Hypothesis (a) is thus fairly natural, and (b) is natural from the standpoint of relating topology and order (clearly it also forces $E=K-K$ ). It is well known that (b) holds if $\mathfrak{I}$ is complete metrizable [5, p. 221, Lemmas 2 or 6, p. 93, Proposition (1.2.6)] and $E=K-K$. For the present purpose it is essential to know that (b) is equivalent to the following condition: if $E^{*}$ is the algebraic dual of $E$, ordered by its positive cone $K=$ $-K^{0}$, then $E^{\prime}$ is full in $E^{*}$, i.e., if $x^{*} \in E^{*}$ is such that there exist $y^{\prime}, z^{\prime}$ in $E^{\prime}$ with $y^{\prime} \leqslant x^{*} \leqslant z^{\prime}$, then $x^{*} \in E^{\prime}$, and moreover the full hull in $E^{*}$ of an equicontinuous set in $E^{\prime}$ is equicontinuous, i.e., if $y^{\prime}$ and $z^{\prime}$ above are restricted to lie in an equicontinuous set, then all the $x^{*}$ satisfying the order relation form an equicontinuous set $([6$, p. 95] and $[7$, p. $14 \mathrm{ff}$.$] ). (Actually$ all we use is the fact that the coarsest topology on $E$ finer than $\sigma\left(E, E^{\prime}\right)$ for which $K$ is conormal is coarser than $\tau\left(E, E^{\prime}\right)$.)

Rather than consider optimum hypotheses for which the implication 
(3) $\Rightarrow$ (1) might hold, let us consider the case of a Fréchet space $E$ ordered by a closed normal generating cone $K$. T. Andô proved in the Banach space case [1, p. 1167, Theorem 2] and A. Goullet de Rugy extended to Fréchet spaces [3, p. 163, Corollary 3.9] that in this case, (3) implies

\section{$E$ has the Riesz decomposition property.}

The point of having (4) available is the following.

Proposition 2. Let E[I] be ordered by a weakly normal generating closed cone $K$ with the Riesz decomposition property. Then positive tensors in $E^{\prime} \otimes E$ have positive traces.

If $E$ is a Banach space ordered by a closed normal generating cone, it is natural to consider the projective tensor product $E^{\prime} \hat{\otimes}_{\pi} E$ in this context; here, in view of [2, Chapter I, p. 114, Proposition 22], the natural topology for $L(E)$ is that of uniform convergence on compacta. Using the results of $[6, \S 1.2$, p. $91 \mathrm{ff}$.$] , one can verify that an order-theoretic variant of the re-$ sult of [2] cited above is available, and one then has an equivalence between $\left(1_{\pi}\right) \quad$ If $\mathrm{t} \in E^{\prime} \quad \hat{\otimes}_{\pi} E$ and $T_{\mathrm{t}} \geqslant 0$, then $\operatorname{tr}(\mathrm{t}) \geqslant 0$, and

$\left(2_{\pi}\right) \quad$ transformation on $E$ belongs to the closure of $P$ in the topology of uniform convergence on compacta.

A metric version of $\left(2_{\pi}\right)$ can also be given. For certain classes of ordered Banach spaces, e.g., those possessing order units or appropriate convexity properties, all those conditions are equivalent (and equivalent to the Riesz decomposition property). E.g., Choquet simplices are characterized by the fact that their spaces of affine functions possess any (or all) of the properties (1), (2), $\left(1_{\pi}\right),\left(2_{\pi}\right)$.

\section{REFERENCES}

1. T. Andô, On fundamental properties of a Banach space with a cone, Pacific J. Math. 12 (1962), 1163-1169. MR 27 \#568.

2. Alexandre Grothendieck, Produits tensoriels topologiques et espaces nucléaires, Mem. Amer. Math. Soc. No. 16 (1955). MR 17, 763.

3. Alain Goullet de Rugy, Un théorème du genre "Andô-Edwards" pour les Fréchets ordonnés normaux, Pacific J. Math. 46 (1973), 155-166.

4. G. J. O. Jameson, Ordered linear spaces, Lecture Notes in Math., vol. 141, Springer-Verlag, Berlin and New York, 1970. 
5. Helmut H. Schaefer, Topological vector spaces, Macmillan, New York, 1966. MR 33 \#1689.

6. Bertram Walsh, Ordered vector sequence spaces and related classes of linear operators, Math. Ann. 206 (1973), 89-138.

7. Yau-Chuen Wong, Lecture notes on nuclear and L-nuclear spaces, Yale University, New Haven, Conn., 1974.

DEPARTMENT OF MATHEMATICS, RUTGERS UNIVERSITY, NEW BRUNSWICK, NEW JERSEY 08903 